\title{
microRNA dysregulation in neurodegenerative diseases: a systematic review
}

Camille A. Juźwik ${ }^{1}$, Sienna S. Drake ${ }^{1}$, Yang $Z_{\text {hang }}{ }^{1}$, Nicolas Paradis-Isler ${ }^{1}$, Alexandra Sylvester ${ }^{1}$, Alexandre Amar-Zifkin ${ }^{2}$, Chelsea Douglas ${ }^{3}$, Barbara Morquette ${ }^{1}$, Craig S. Moore ${ }^{4}$ Alyson Fournier ${ }^{1}$

(1) McGill University, Montréal Neurological Institute, 3801 University Street, room BT111, Montréal, QC, H3A 2B4, Canada

(2) McGill University Health Centre- Medical Libraries, 3801 University Street, Montréal, QC, H3A 2B4, Canada

(3) Program Manager, Plotly Technologies Inc, 5555 Gaspe Avenue \#118, Montréal, QC, H2T 2A3, Canada

(4) Division of BioMedical Sciences Faculty of Medicine, Memorial University of Newfoundland, St. John's, NL, Canada

\section{Email Contact:}

Camille A. Juźwik: camille.juzwik@mail.mcgill.ca

Sienna S. Drake: sienna.drake@mail.mcgill.ca

Yang Zhang: yang.zhang5@mail.mcgill.ca

Nicolas Paradis-Isler: nicolas.paradis-isler@mcgill.ca

Alexandra Sylvester: alexandra.sylvester@queensu.ca

Alexandre Amar-Zifkin: alex.amar@muhc.mcgill.ca

Chelsea Douglas: cldoug1@gmail.com

Barbara Morquette: barbara.morquette@mcgill.ca

Craig S. Moore: craig.moore@mun.ca

Alyson Fournier: alyson.fournier@mcgill.ca 


\section{Introduction}

Neurodegenerative diseases are defined by the progressive deterioration of neuronal structure and function eventually leading to neuronal loss that is thought to underlie most of the neurological impairments 1. In addition to neuronal cell dysfunction and death, neurodegenerative diseases often share common cellular mechanisms and histopathological features regardless of their etiology ${ }^{2}$. For instance, aberrant protein processing, trafficking and aggregation in neural cells are observed in Alzheimer's disease (AD), amyotrophic lateral sclerosis (ALS), Huntington's disease (HD), Parkinson's disease (PD), prion diseases as well as in other neurodegenerative diseases ${ }^{3-5}$. This disruption in cellular protein homeostasis is often associated with extracellular plaque build-up that induce local inflammation through astrocyte and microglia activation ${ }^{2,3,6-8}$. Aberrant RNA metabolism is another common feature for several neurodegenerative diseases: ALS, ataxia, HD and myotonic dystrophy (DM) ${ }^{9-11}$. Furthermore, dysfunctional glutamate neurotransmission leading to neurotoxicity and oxidative injury are also a recurring theme in neurodegenerative diseases, including epilepsy 2, 12-15. Shared pathophysiological processes in multiple neurodegenerative diseases raises the possibility that identifying common molecular regulation across various diseases may lead to approaches for therapies, which would be effective for multiple indications.

MicroRNAs (miRNAs) regulate hubs of gene expression and are dysregulated in many neurodegenerative diseases and their animal models ${ }^{16-20}$. miRNA biogenesis is a two-step cleavage process where a hairpin structure is made progressively shorter; the initial cleavage by ribonuclease Drosha and Dgcr8, and the second cleavage event by Dicer alone to form a miRNA duplex ${ }^{21}$. The duplex is loaded onto an Argonaute protein to form the core of the miRNAinduced silencing complex (miRISC), where a passenger strand of the duplex is ejected. The miRISC binds to the 3'-untranslated region (3'UTR) of target mRNA strands, leading to the degradation or translational repression of mRNAs. Disruption of the miRNA biogenesis machinery has been used to demonstrate the critical role of miRNAs in the central nervous system (CNS) ${ }^{22-34}$. Specifically, deletion of Dicer in different brain regions leads to brain atrophy, neurodegeneration, gliosis, locomotor deficits, and shortened lifespans 23, 25, 27, 28, 30-32. Similarly, retinal deletion of Dicer or Dgcr8 lead to retinal degeneration and loss of visual function 22, 24, 26, 29, 33, 34 . Decreased Dicer expression has also been observed in patients with advanced aged-macular degeneration (AMD), epilepsy, and multiple sclerosis (MS) ${ }^{29,}{ }^{35-37}$. Animal models of epilepsy and MS also exhibit decreased Dicer expression ${ }^{37,38}$. Disruption of the miRNA-processing machinery provides proof-of-principle evidence that miRNAs are essential to nervous system function and integrity, and that disruption of miRNA expression could contribute to deficits identified in some neurodegenerative diseases.

Reviews of the literature describe the disruption of miRNA expression within specific neurodegenerative diseases but miRNA regulation across neurodegenerative diseases has not been systematically reviewed ${ }^{39-41}$. Identifying shared miRNA dysregulation across neurodegenerative diseases may lend insight into the conserved molecular pathways affected 
during disease and potentially point towards novel targets for creating therapies aimed at general degenerative mechanisms involved in many neurodegenerative diseases. Here we perform a nonbiased systematic review of studies identifying miRNA dysregulation in neurodegenerative diseases to facilitate identification of shared patterns of miRNA dysregulation across multiple neurodegenerative diseases. We recorded miRNA expression from articles that report miRNA expression spanning 12 neurodegenerative diseases and their animal models: AD, amyotrophic lateral sclerosis (ALS), AMD, ataxia, non-AD related dementia, DM, epilepsy, glaucoma, HD, MS, PD, and prion disorders. We describe seven individual miRNAs and one miRNA family occurring frequently within and across neurodegenerative diseases: miR-9-5p, miR-21-5p, miR124-3p, miR-132-3p, miR-146a-5p, miR-155-5p, miR-223-3p, and the miR-29 family. Interestingly, three of these miRNAs were frequently upregulated in neurodegenerative diseases, while the other seven miRNAs did not exhibit a conserved direction of dysregulation. We also report that these miRNAs have roles in both the immune system and CNS, suggesting inflammation as a major component of neurodegenerative disease. 


\section{Methods}

Search strategies- A systematic and comprehensive search of the literature was conducted by a librarian (AA-Z) to retrieve articles discussing neurodegenerative conditions and miRNA. A search strategy was developed for Medline via Ovid and adapted to other databases: Embase, PSYCinfo and Biosis Previews, all also via Ovid. The Medline search strategy searched in controlled vocabulary where possible, along with text in the title, abstract, or author-supplied keyword fields. Searches conducted subsequent to Medline excluded the articles already found. The searches were initially run on 2017-07-13 and were updated with additional terms on 201711-07 and 2019-03-22. The updated Medline strategy is included in Supplementary File 1. Reference lists of included studies were also evaluated. Search terms were selected to be inclusive of all neurodegenerative diseases.

Data management and screening- Results of the literature searches (6421 publications) were imported into an Endnote library. Duplicate publications were removed prior to review. Two independent reviewers assessed the eligibility of articles using a policy of liberal acceleration: only one reviewer's approval was necessary to advance a publication to the next stage of screening, but both reviewers had to agree on the exclusion of a publication, as previously described for Preferred Reporting Items for Systematic Reviews and Meta-Analyses (PRISMA) 42,43 . This strategy was chosen to ensure that every rejected article was validated as unrelated to the topic of this review by a second reviewer.

Articles went through four rounds of evaluation (Fig. 1). The first two rounds assessed the relevance of the article to the review topic (miRNA and neurodegenerative disease) and the type of article (review, conference abstract, primary research article, etc.) based on the information in their title and abstract. At the title and abstract stages, articles were excluded if they were not primary research articles, or if they did not refer to either miRNA or neurodegenerative disease.

At the manuscript stage papers were evaluated using a more rigorous set of exclusion criteria: not a primary research article; no in vivo or ex vivo results; no reporting of differential expression data of mature miRNA or only reporting differential miRNA expression data taken from previous studies or from online repositories; not related to neurodegenerative disease; a duplicate; and/or inaccessible. These criteria were chosen to identify papers that reported new miRNA regulation data of biological material from neurodegenerative diseases and their models. Following the manuscript evaluation, papers were sorted into categories based on the neurodegenerative diseases studied. Any neurodegenerative disease category with fewer than 10 papers were excluded from further analysis as this was determined to be below the threshold for synthesis and analysis. This resulted in 641 accepted articles making up 12 disease categories: AD, ALS, AMD, ataxia, dementia, DM, epilepsy, glaucoma, HD, MS, PD, and prion disorders (Fig. 1).

Next, we wanted to determine what miRNAs were frequently dysregulated across these diseases. For each of the 641 accepted manuscripts, we recorded the miRNAs that were reported as significantly dysregulated, and the direction of dysregulation relative to control in a neurodegenerative disease and/or its animal model. We also list the identify of the profiled tissue 
or bodily fluid (Supplementary File 2). When miRNAs were identified by microarray or nextgeneration sequencing, only miRNA regulation that was validated by qPCR was recorded. Studies incorporating miRNA overexpression or knockdown in an animal model of neurodegenerative disease were also recorded. miRNA annotation was confirmed using the miRbase Database ${ }^{44}$. We recorded a total of 2318 dysregulated miRNAs identified in the 641 manuscripts. We then assessed which miRNAs occurred in over half (7/12) of the disease categories and found 52 miRNAs that fit this criterion. Of these 52 miRNAs, some also occurred more frequently within the diseases. In order to identify the miRNAs occurring most frequently both across and within neurodegenerative diseases, we applied cut-offs per disease category based on the number of publications (Supplementary File 2, tab 1; Fig. 1). This identified 7 individual miRNAs that are miR-9-5p, miR-21-5p, miR-124-3p, miR-132-3p, miR-146a-5p, miR-155-5p, miR-223-3p. We also identified three miRNA families that occurred within our 52 miRNAs. From those three miRNA families, the miR-29 family occurred most frequently, and it was included for analysis with the 7 individual miRNAs.

Heat map- To determine if a miRNA was regulated in a distinct direction within our 10 assessed neurodegenerative diseases, either upregulated or downregulated during disease compared to control, we generated a heat map synthesizing their expression. If a study assessed the expression at multiple stages of diseases, or within multiple tissue, this was included as an additional data point. For each disease when a miRNA was upregulated in a distinct tissue, it was assigned 1, and if downregulated it was assigned -1 . The sum was taken for each miRNA for a disease, and then divided by the amount of times it was identified as differentially dysregulated to determine a rank. If a miRNA has a rank closer to 1 , this implies that it was mainly upregulated. If a miRNA has a rank closer to -1 , this implies it was mainly downregulated. If a miRNA has a rank close to 0 , this implies that it was reported as significantly upregulated and downregulated in an approximately equal number of tissues. Data that compared miRNA expression between patients treated with a disease-modifying therapies (DMTs) versus baseline were not included; nor were animal studies where the miRNA expression was manipulated. Data was compiled into an CSV file read by a python script with pandas (http://pandas/pydata.org/) to create a dataframe. We used Plotly's python API (http://plot.ly/) to create a heatmap from the dataframe ${ }^{45}$. 


\section{Results}

Our initial search strategy of the systematic review resulted in a sum of 6421 publications, of which 989 were reviewed at the manuscript level (Fig. 1). Diseases that had fewer than 10 manuscripts were excluded as they were below our threshold for synthesis. This resulted with 641 manuscripts covering 12 disease categories: AD, ALS, AMD, ataxia, dementia, DM, epilepsy, glaucoma, HD, MS, PD, and prion disorders (Fig. 1).

To identify frequently dysregulated miRNAs spanning multiple neurodegenerative diseases, for each of the final 641 analyzed manuscripts, we identified the profiled tissue and if the miRNA was significantly upregulated or downregulated relative to control in neurodegenerative disease and/or its animal model. Animal models are an important consideration for some neurodegenerative diseases such as AMD, ataxia, dementia, glaucoma, epilepsy, HD, and prion disorders where there are few studies investigating dysregulation of miRNA in human material. All accepted manuscripts sorted by disease and denoting manuscripts that profiled miRNA expression in multiple neurodegenerative diseases are presented in Supplementary File 2. Studies incorporating miRNA overexpression or knockdown in an animal model of neurodegenerative disease were also recorded.

Using this set of 641 manuscripts we identified 10 miRNAs that were differentially regulated in at least $50 \%$ of the neurodegenerative diseases and/or their animal models that were also frequently dysregulated within the individual disease categories: miR-9-5p, miR-21-5p, miR29a-3p, miR-29b-3p, miR-29c-3p, miR-124-3p, miR-132-3p, miR-146a-5p, miR-155-5p, and miR-223-3p. We reviewed the manuscripts describing regulation of these miRNAs during neurodegenerative diseases in greater detail and summarized the miRNA expression profiles in Table 1. We found that miRNA expression data from the animal models did not confound the human data, but rather supported it. To visualize the expression of these 10 miRNAs across neurodegenerative diseases and their animal models we generated a heat map to represent changes in expression (Fig. 2). A limitation of these studies is the small number of studies for some miRNA in individual diseases. For example, miR-29b-3p and miR-132-3p are reported in a single study for AMD as upregulated, resulting in a score of 1 on the heat map.

To generate hypotheses about how these 10 frequently dysregulated miRNAs may contribute to neurodegenerative disease we thoroughly reviewed the papers and their known mechanism of action. Figure 3 summarizes the validated targets and pathways identified by these studies, breaking down the pathways by immune and neural components. 


\section{Upregulated miRNAs}

Within the top ten miRNAs, we found the miRNAs miR-146a-5p, miR-155-5p, and miR-223-3p to be generally upregulated across the 12 investigated diseases ("miRNA sum" $\geq 0.5$; Fig. 2) (Table 1). Upregulation of these miRNAs across neurodegenerative disease raises the possibility that they may contribute to common mechanisms underlying disease pathogenesis and the possibility that they are upregulated as a response to neurodegeneration itself. Here we discuss the known functions of these three miRNAs in more detail.

miR-155-5p

miR-155-5p is described as a master regulator of the immune response, specifically driving myeloid cell polarization to a pro-inflammatory state ${ }^{46,47}$. Deletion of miR-155 prolonged survival in SOD ${ }^{\mathrm{G} 93 \mathrm{~A}}$ mice, prevented Experimental Autoimmune Encephalomyelitis (EAE) induction, and attenuated microgliosis and neuronal loss in an $\alpha$-synuclein-driven PD model ${ }^{48-56}$. In the PD model, miR-155-5p deletion was associated with a reduction of MHCII expression in microglia and attenuated iNOS upregulation in response to $\alpha$-synuclein ${ }^{48}$. Improvements in the SOD $1^{\mathrm{G} 93 \mathrm{~A}}$ and EAE models upon miR-155 deletion were associated with decreased expression of proinflammatory cytokines interferon- $\gamma$ (IFN- $\gamma$ ) and interleukin-17 (IL-17) (Fig. 3) ${ }^{49-56}$. miR155 deletion also causes increased expression of targets that block myeloid cell proliferation and survival, Th17 development, oxidative stress, and blood brain barrier (BBB) permeability ${ }^{53,56-58}$. Increased miR-155-5p expression across the profiled neurodegenerative diseases, and its wellknown roles in promoting neuroinflammation raises the possibility that increased miR-155-5p expression may exacerbate neurodegenerative disease pathology via an inflammatory mechanism.

$\operatorname{miR}-223-3 p$

miR-223-3p is a well-characterized hematopoietic miRNA, regulating myeloid cell and granulocyte differentiation, and dendritic cell activation ${ }^{59-62}$. In MS and EAE, miR-223-3p upregulation is associated with pathogenic polarization of immune cells and increased inflammation. miR-223 -/ EAE animals possess diminished populations of pathogenic Th1 and Th17 cells, due to a larger population of myeloid-derived regulatory cells and reduced dendritic cell activation ${ }^{62-64}$. These lines of evidence suggest that upregulation of miR-223-3p within immune components may be deleterious in neurodegenerative disease. However, early work characterizing miR-223-3p suggest that the roles of miR-223-3p may be more nuanced since both overexpression or deficiency of miR-223 leads to granulocyte overproduction and widespread inflammation ${ }^{59,60}$. miR-223-3p targets and inhibits components within the NF- $\kappa \beta$ pathway, including TRAF6, Tab1, and Culla/b, which are part of the canonical pathway; and $\mathrm{IKK} \alpha$, which controls both canonical and non-canonical activation of NF- $\mathrm{KB}{ }^{65}$, 66 . Through suppression of the canonical NF- $\mathrm{KB}$ pathway, miR-223-3p expression is shown to dampen neutrophil activation, suggesting anti-inflammatory effects ${ }^{66}$. Conversely, miR-223-3p also targets IKK $\alpha$, an anti-inflammatory factor that may prevent spontaneous activation of 
macrophages, thus promoting inflammation ${ }^{65}$. The involvement of miR-223-3p in both the proand anti-inflammatory NF- $\mathrm{kB}$ cascades demonstrates the divergence in its immune regulatory roles.

In addition, miR-223-3p appears to promote neural repair and regeneration. In an animal model of ischemia, miR-223 -/- mice showed contextual memory deficits, enhanced excitotoxicity, and neuronal death ${ }^{67}$. miR-223-3p overexpression blocked these effects by targeting GluR2 and NR2B, AMPA receptor and NMDA receptor subunits, respectively (Fig. 3). Similarly, miR-223 overexpression in the retina and optic nerve blocked the formation of EAE-driven pathological axonal swellings, attributed to decreased excitotoxicity by reduced GluR2 and NR2B expression 68. In models of regeneration, miR-223-3p is upregulated, specifically in mouse DRGs post sciatic nerve lesion, and in the regenerating optic nerve of zebrafish ${ }^{69,70}$. These results suggest miR-223-3p may play a direct role in the neuronal response to injury.

miR-146a-5p

miR-146a-5p is also a major regulator of the NF- $\mathrm{kB}$ pathway ${ }^{71}$. The pre-miR-146a gene is positively regulated by $\mathrm{NF}-\mathrm{\kappa B}$ transcription factor and the mature miRNA-146a-5p product operates in a negative feedback loop by inhibiting IRAK1 and TRAF6, two upstream NF- $\kappa B$ signaling components (Fig. 3) ${ }^{71}$. This regulatory loop operates as a brake against rampant myeloid-driven inflammation. Indeed, $m i R-146 a-/$ animals had worsened motor pathology with a massive 10-fold increase in myeloid cell proliferation and T-cell activation caused by relief of inhibition on IRAK1 and TRAF6 ${ }^{72,}{ }^{73}$. miR-146a-5p targeting of IRAK1 and TRAF6 also reduces T-cell adhesion in vitro, indicating that miR-146a-5p may impair the ability of immune cells to cross the BBB ${ }^{74}$. These functions of support a model whereby upregulation of miR$146 \mathrm{a}-5 \mathrm{p}$ may play a neuro-protective role in neurodegenerative disease. This is further supported by a report that miR-146a-5p overexpression promotes remyelination of axons in the cuprizone model of demyelination ${ }^{75}$.

miR-146a-5p overexpression can also indirectly lead to the promotion of pro-inflammatory noncanonical NF- $\kappa B$ signaling ${ }^{76}$. Specifically, downregulation of TRAF6 and IRAK1 of the NF- $\mathrm{kB}$ pathway by miR-146a-5p overexpression is compensated by an upregulation in IRAK2, in turn activating myeloid cells and promoting cytokine release that perpetuates TLR-IL-1R-mediated $\mathrm{NF}-\mathrm{kB}$ activation, as shown in AD models ${ }^{76}$. Thus, miR-146a-5p may be a protective response to disease by limiting NF- $\mathrm{KB}$ signaling and promoting remyelination. However, the complexity of the negative feedback loop between miR-146a-5p and NF- $\mathrm{kB}$ suggests that increased levels of miR-146a-5p can indirectly contribute to other pro-inflammatory responses. Increased levels of miR-146a-5p coincide with increased AD clinical scores ${ }^{77}$; and in AD mouse models, miR-146a$5 p$ positively correlates with clusters of activated microglia ${ }^{78}$. In EAE, an animal model of MS, stress-exposure that exacerbated disease also further increased miR-146a-5p expression ${ }^{73,79}$. Thus miR-146a-5p upregulation may have a dichotomous role with both pro-inflammatory and anti-inflammatory responses with divergent effects in neurodegenerative disease progression. 


\section{Mixed Regulation}

The remaining miRNAs, miR-9-5p, miR-21-5p, the miR-29 family, miR-124-3p, and miR-132$3 p$ demonstrate mixed level of expressions across tissue and animal models of the 12 investigated neurodegenerative diseases $(-0.5<$ "miRNA sum" $<0.5$; Figure 2$)$. Their regulation and functions are further described below.

miR-9-5p

Many miR-9-5p functions are related to CNS development, including the proliferation and differentiation of neural stem cells, and levels remain enriched throughout adulthood (Fig. 3) ${ }^{80}$, 81 82. In SOD1 ${ }^{\mathrm{G} 93 \mathrm{~A}}$ mice, an ALS model, degeneration of motor neurons promotes the proliferation and differentiation of neuronal progenitors. This effect has been proposed as a compensatory neurogenesis response against motor neuron loss ${ }^{83,84}$. Elevated levels of miR-9$5 \mathrm{p}$ within CNS tissue may reflect the accelerated proliferation and differentiation of progenitors ${ }^{85}, 86$. Accordingly, miR-9-5p upregulation within CNS tissue is also negatively correlated with STAT3 activation, a factor known to inhibit neuronal cell fate ${ }^{85,87}$. The role of miR-9-5p in modulating neuronal fate in neurodegenerative disease is further supported by miR-9-5p targeting transcription factor REST ${ }^{88}$. REST suppresses neural fate and promotes miR-9-5p expression, which targets and inhibits REST expression in a negative feedback loop. This homeostasis is disrupted in HD, contributing to disease pathogenesis ${ }^{88}$. Neurodegenerative diseases $\mathrm{AD}, \mathrm{HD}, \mathrm{MS}$ and $\mathrm{PD}$ also show signs of altered neurogenesis ${ }^{89-92}$. This raises the interesting possibility that dysregulation of miR-9-5p and subsequent changes in neuronal progenitor populations may be a common altered mechanism between neurodegenerative diseases.

$m i R-21-5 p$

In animal studies modelling aspects of MS (EAE) and PD (MPTP), miR-21-5p downregulation appeared to be neuroprotective ${ }^{93-95}$. miR-21-5p expression is downregulated in an EAE-resistant rat strain, and $m i R-21$ - $/$ mice were resistant to EAE induction and exhibited defects in Th17 differentiation (Fig. 3) ${ }^{94,95}$. In MPTP-treated mice, miR-21-5p knockdown resulted in increased neuronal survival ${ }^{93}$. This neuroprotective effect was mediated by increasing the expression of the miR-21-5p target LAMP2A. LAMP2A promoted autophagy of $\alpha$-synuclein, thereby limiting pathogenic aggregation ${ }^{93}$. miR-21-5p downregulation also relieved its target PPAR $\alpha$, a transcription factor that promotes the expression of neuroprotective factors such as brain-derived and glial derived neurotrophic factors (BDNF and GDNF, respectively), and limited the expression of neuroinflammatory factors including NF- $\mathrm{kB}{ }^{96}$. These studies suggest that miR-21$5 \mathrm{p}$ inhibition dampens the inflammatory response and promotes neuroprotection.

Conversely, miR-21-5p was often downregulated in AD patients and animal models ${ }^{97-100}$. In an animal model of $\mathrm{AD}$, overexpression of miR-21-5p promoted functional recovery ${ }^{101}$. Likewise, overexpression of miR-21-5p in a mouse model of glaucoma, induced by increased intraocular 
pressure (IOP), prevented loss of retinal neurons ${ }^{102}$. Increased IOP in the glaucoma model is associated with increased $A \beta$-deposition in apoptotic retinal neurons, which can be targeted to reduce disease pathogenesis ${ }^{103}$. Thus, in AD and glaucoma models, miR-21-5p dysregulation is likely related to $A \beta$ pathology. Ultimately, the dichotomous dysregulation of miR-21-5p points to a complex role in neurodegenerative disease and additional research in its functional contributions to disease would offer clarity.

miR-29 family

The miR-29 family consists of two miRNA clusters, miR-29ab1 and ${ }^{104}$. The clusters produce miR-29a, miR-29b and miR-29c; these miRNAs share the same seed sequences and thus the ability to target the same genes. Animal studies suggest miR-29 is necessary for proper motor function. miR-29ab loss-of-function shows severe motor impairment ${ }^{105,106}$. Disease modifying treatments of both PD and MS patients resulted in miR-29a-3p upregulation relative to untreated controls $^{107-109}$. This suggests that these disease-modifying treatments (DMTs) may aid in restoring normal expression of miR-29a-3p. However, challenging this are experiments showing that miR-29ab1 deletion in EAE mice led to an improvement in the EAE disease course ${ }^{104}$. This could be a result of a negative feedback loop formed by miR-29 and IFN- $\gamma$, where IFN- $\gamma$ induces pathogenic Th1 biology in EAE mice (Fig. 3) ${ }^{104,110}$. This falls in line with decreased miR-29-a$3 p$ and miR-29c-3p in the peripheral blood mononuclear cells (PBMCs) of MS patients following treatment with the DMT IFN- $\beta^{111}$.

miR-124-3p

miR-124-3p expression is predominantly expressed in the CNS and most articles profiled its dysregulation in CNS tissue (Table 1) ${ }^{112}$. Overexpression of miR-124-3p led to symptomatic recovery in animal models of AD, AMD, glaucoma, HD, MS, and PD ${ }^{112-120}$. Functionally, miR124-3p has been implicated in apoptotic signaling, autophagy, neurogenesis, glutamate signaling, and immune modulation (Fig. 3). miR-124-3p overexpression in these animal models appears to regulate these functions to limit or prevent disease pathogenesis.

In an AD model, miR-124 overexpression reduced expression of BACE1; decreased pathological tau; restored autophagy function by reducing Bax; and restored or improved behavioural outputs such as memory $112,113,119$. In a PD animal model, miR-124-3p directly targeted and inhibited Bim, a protein that mediates translocation of Bax to both mitochondrial and lysosomal membranes, mediating both apoptotic and autophagic pathways, respectively ${ }^{121}$. Thus, upregulation of miR-124 may inhibit apoptotic processes through inhibition of Bim and indirectly through Bax.

In a PD model, miR-124-3p delivery to the subventricular zone by nanoparticles enhanced neurogenesis and neural cell differentiation by targeting cell-fate proteins Sox9 and Jagged1 (Fig. 3) ${ }^{114}$. In vitro these nanoparticles also promoted axonogenesis through modulation of the c-Jun N-terminal kinase (JNK) pathway. Likewise, in ALS and HD mouse models, Sox9 
downregulation was associated with increased miR-124-3p ${ }^{85,116}$. Similar to miR-9-5p, this suggests miR-124-3p may enhance neurogenesis and differentiation of NPCs, perhaps to compensate for neuronal loss within ALS and HD mouse models.

miR-124-3p is also suggested to limit glutamate excitotoxicity by targeting AMPA receptors (AMPAR) (Fig. 3) ${ }^{122-124}$. In an AD mouse model, miR-124-3p upregulation in the brain was associated with a decrease in target PTPN1 ${ }^{122}$. PTPN1 inhibition lead to decreased AMPAR membrane-insertion, resulting in AMPAR downregulation. miR-124-3p can also directly target GluR2, an AMPAR subunit ${ }^{123}$. This was observed in an animal model of dementia and in demyelinated MS lesions ${ }^{123,}{ }^{124}$. In dementia, miR-124-3p overexpression or GluR2 knockdown rescued behavioural deficits; and in the lesions of human MS and in demyelinated mouse hippocampi, increased miR-124-3p was associated with AMPAR downregulation ${ }^{123,}{ }^{124}$. Remyelination of mouse hippocampus reversed these changes. These findings suggest a neuroprotective mechanism in which miR-124-3p downregulates AMPAR to reduce glutamate excitotoxicity in regions of demyelination.

Finally, miR-124-3p was identified to have an immunomodulatory effect both in MS and PD, where its overexpression reduced macrophage/microglia activation to limit disease progression (Fig. 3) ${ }^{115,120}$. In sum, overexpression of miR-124-3p appears to predominantly promote functional repair in various animal models of neurodegenerative disease.

miR-132-3p

miR-132 and miR-212 form a cluster, where the two miRNAs have similar sequences and thus seed regions; however, miR-132 is the major functional species in the brain ${ }^{125}$. miR-132-3p was predominantly downregulated in CNS tissue (Table 1), suggesting it may be required for proper CNS function. miR-132-3p expression is promoted by CREB - a transcription factor typically associated with promotion of neurotrophic factors - and is downregulated by REST (Fig. 3$)^{126}$. Through CREB, miR-132-3p promotes neurite outgrowth, dendritic growth, and maintenance of the circadian clock ${ }^{127-129}$. The importance of miR-132-3p has been demonstrated through lossof-function experiments. miR-132-3p deletion promotes apoptosis in neurons ${ }^{130}$. AD mouse models crossed with miR-132/212 -/- mice display worsened long-term memory, enhanced A $\beta$ burden, and increased tau pathology; where multiple genes of the tau subnetwork are miR-1323p targets $125,131,132$.

EAE mice treated with anti-inflammatory agent tetrachlordodibenzo-p-dioxin (TCDD) exhibited decreased clinical deficits and these treatment effects were lost with miR-132 loss-of-function, thus demonstrating that miR-132-3p is involved in anti-inflammatory attenuation of EAE by Th2-promoting TCDD ${ }^{133}$. TCDD upregulation of miR-132-3p decreases target acetylcholinesterase, relieving hydrolysis of acetylcholine, a key suppressor of pro-inflammatory cytokines ${ }^{126,133}$. However, like TCDD, proinflammatory FICZ also promoted miR-132-3p upregulation ${ }^{133,}{ }^{134}$. miR-132-3p's protective roles in inflammatory modulation is further 
complicated by its involvement in Th1 and Th17 immune cells. miR-132/212 -/- mice showed a resistance to EAE induction, affiliated with lower frequencies of Th1 and Th17 cells (Fig. 3) ${ }^{134}$. Th1/Th17 dominant paradigms, expressing higher IL-17, IFN- $\gamma$, and TNF- $\alpha$ levels, have elevated miR-132-3p ${ }^{135-137}$. These results demonstrate that miR-132-3p has context-dependent roles in both anti-inflammatory and pro-inflammatory pathways.

\section{Disease trends}

Interestingly, global trends for the predominant miRNAs taken collectively were uncovered within some disease categories. In the AMD, MS and prion disease categories, the predominant miRNAs surveyed were more frequently reported to be upregulated ("Disease sum" $\geq 0.5$ ). Inversely, in DM and HD disease categories the different miRNAs surveyed were predominantly reported to be downregulated ("Disease sum" $\leq-0.5$; Figure 2). For the remaining ( 7 out of 12) disease categories, no clear global trends emerge out of the reports for the miRNAs surveyed ($0.5<$ "Disease sum" $<0.5$; Figure 2). Whether this suggests anything about the pathogenesis of the disease is unclear. It is possible that there are disease mechanisms that generally interfere with miRNA homeostasis. 


\section{Discussion}

MiRNAs are pervasive post-transcriptional regulators and their involvement in neurodegenerative disorders is supported by a vast literature. With this systematic review of the literature, we identified miRNAs that are commonly dysregulated across neurodegenerative diseases. We identified miR-9-5p, miR-21-5p, the miR-29 family, miR-124-3p, miR-132-3p, miR-146a-5p, miR-155-5p, and miR-223-3p as the miRNAs predominantly reported to be dysregulated in 12 categories of neurodegenerative disease and related animal models (Fig.1).

One of the main findings of this review is that three miRNAs, miR-146a-5p, miR-155-5p, and miR-223-3p, are predominantly upregulated across the neurodegenerative disease categories. However, the other miRNAs identified as predominant in the reviewed literature are either subject to conflicting reports within disease categories or exhibit opposing trends between categories. We also identified directional regulation of the predominant miRNAs within diseases. MS, prion, and AMD disease categories demonstrated a general upregulation of the predominant miRNA species. Conversely, HD and DM demonstrated an overall downregulation of these species. This may indicate specific disease mechanisms have overarching effects on miRNA homeostasis. Indeed, one can speculate that HD and DM, both being trinucleotide repeat disorders resulting in aberrant RNA and protein production and degradation, may result in similar interference in miRNA homeostasis. However, since miRNAs are frequently studied in isolation, there is very little known about the mechanisms behind the dysregulation of multiple miRNAs in neurodegenerative diseases. Since the scope of this article was limited to the predominant miRNAs across neurodegenerative diseases as well, we cannot say whether this dysregulation is conserved when looking at the whole list of miRNAs dysregulated within a specific disease.

We also summarized pathways known to be targeted by the miRNAs commonly dysregulated in neurodegenerative diseases (Fig. 4). There lies important functional overlap between these miRNAs in regulating differing cellular pathways with multiple miRNAs often converging on the same pathways. Pathways targeted by multiple miRNAs include A $\beta$ genesis, regulation of AMPAR subunits, autophagy homeostasis, apoptosis, microglial activation, NF- $\kappa \mathrm{B}$ signaling, $\mathrm{BBB}$ maintenance, and neurogenesis. The largest shared convergence occurs with A $\beta$ genesis, where miR-146a-5p, the miR-29 family, miR-124-3p, and miR-9-5p all limit A $\beta$ genesis. miR-9$5 p$, miR-124-3p, miR-29a-3p, miR-29b-3p, and miR-29c-3p target BACE1. Target site cooperation is a fundamental characteristic of miRNA-mediated silencing suggesting these miRNAs are likely to be functioning synergistically to facilitate repression of their shared targets ${ }^{138,139}$. We also see miRNAs converging on the same pathways but with alternate outputs. For example, miR-29 family members, miR-223-3p, miR-155-5p, and miR-132-3p all regulate $\mathrm{T}$ cell activation and proliferation. However, while miR-223-3p and miR-155-5p promote a Th1/Th17 profile, the miR-29 family inhibits Th1 promotion by blocking IFN- $\gamma$ signaling. miR132-3p promotes or blocks development of a Th1/Th17 inflammatory milieu depending on the stimuli. We suggest that these miRNAs likely function cooperatively to directly repress 
individual targets, as well as indirectly by targeting different mRNAs from within the same pathways.

In summarizing the known functions of these predominant miRNAs, we identified roles for each miRNA in regulating distinct neuronal and immune aspects of disease. A major pathway intersecting both immune system regulation and the CNS was the NF-kB pathway (Fig. 4). miR$146 \mathrm{a}-5 \mathrm{p}$ and miR-223-3p targeted multiple components of this pathway regulating both proinflammatory and anti-inflammatory responses ${ }^{140,141}$. However, the other predominant miRNAs could contribute indirectly by regulating promoters of NF- $\kappa B$ signaling such as $A \beta$, excess glutamate, and inflammatory cytokines ${ }^{141}$. Non-canonical NF- $\mathrm{KB}$ signaling in microglia mediates the production of more inflammatory cytokines and neurotoxic molecules such as glutamate and ROS, which only feeds back into pathological microglial activation ${ }^{141,}{ }^{142}$. Microglia represent the innate immune cells of the CNS contributing to both defence and maintenance of the CNS 143. We identified miR-9-5p, miR-124-3p, miR-132-3p, miR-155-5p, and miR-223-3p as regulators of microglial activation. Our systematic review thus complements previous reports of miR-9-5p, miR-124-3p, miR-132-3p, miR-146a-5p, and miR-155-5p as "NeurimmiRs", which are defined as gatekeepers of both the nervous and immune system ${ }^{144}$. We suggest that miR-21$5 p$, the miR-29 family, and miR-223-3p to be similarly considered NeurimmiRs as they also simultaneously modulate immune cell activation and neuronal function. Many of the predominant miRNAs also were involved in T-cell differentiation and activation further emphasizing their immune roles. Overall these results suggest there is a robust immune response during neurodegenerative disease that may be underestimated in the literature.

In summary, we identified trending miRNAs across neurodegenerative disease. We noted overlapping functions for these miRNAs, suggesting that they work in concert across the diseases. We also noted a strong role for each miRNA in both the neuronal and immune compartments during disease. There are many ways to parse miRNA dysregulation within and across neurodegenerative diseases. By conducting a systematic review of articles discussing miRNA dysregulation in neurodegenerative disease, we have made available a wealth of information to be further exploited in the interest of identifying miRNA dysregulation within or across neurodegenerative disease (Supplementary File 2). Our analysis supports the hypothesis that the identification and future characterization of miRNAs involved in pathological mechanisms common to multiple neurodegenerative diseases may help improve our understanding of commonalities in disease pathogenesis and may aid in novel hypotheses relating to cross cutting DMTs. 


\section{Figures}

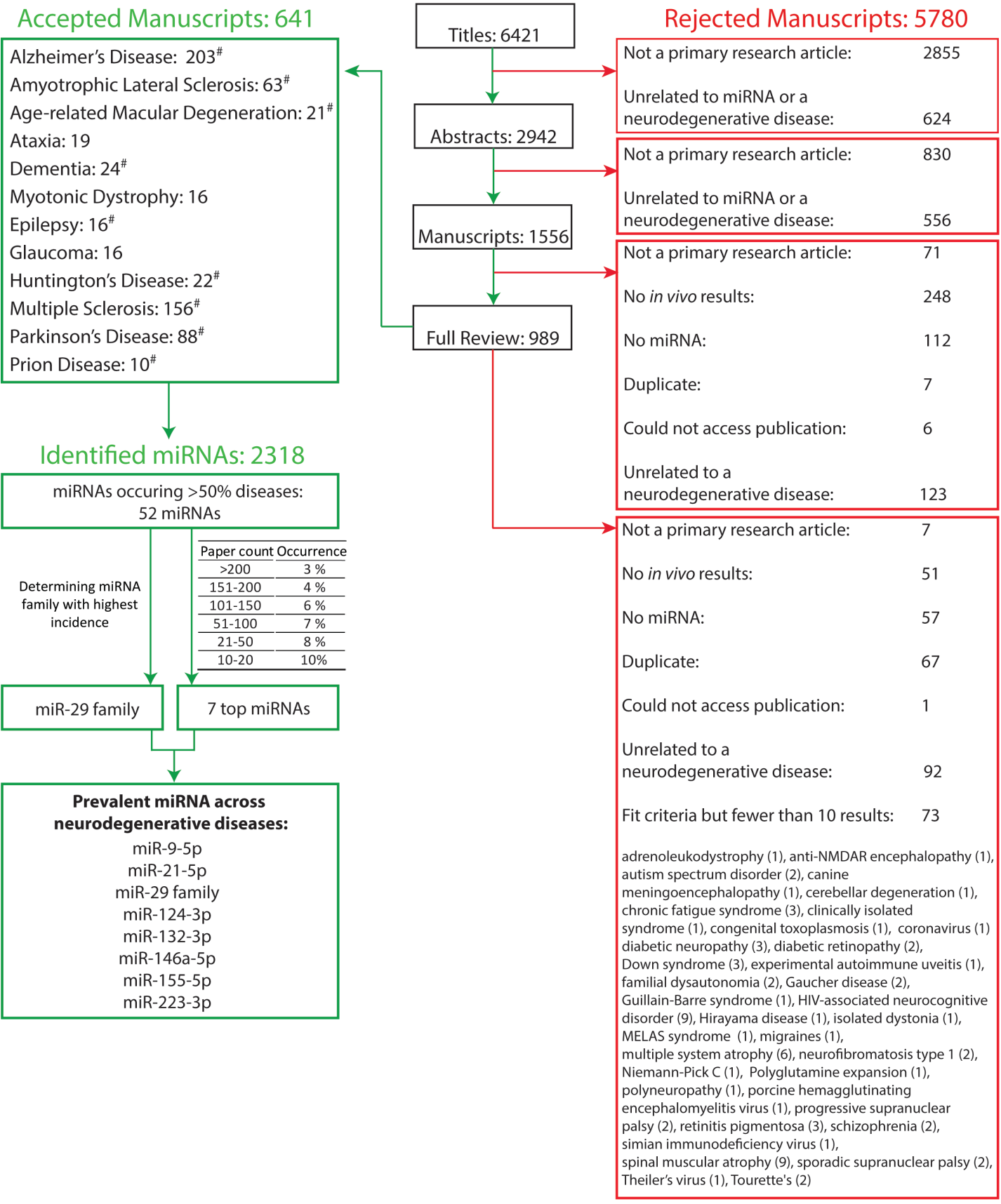

Figure 1. PRISMA flowchart of the systematically reviewed manuscripts with differential miRNA expression in neurodegenerative disease and their animal models. Initial library searches identified 6421 articles. Accepted papers were categorized based on the neurodegenerative disease, and disease categories with less than 10 results were excluded from the review. Diseases marked with \# indicates shared papers between assessed diseases. Supplementary File 2 contains all accepted manuscripts. 


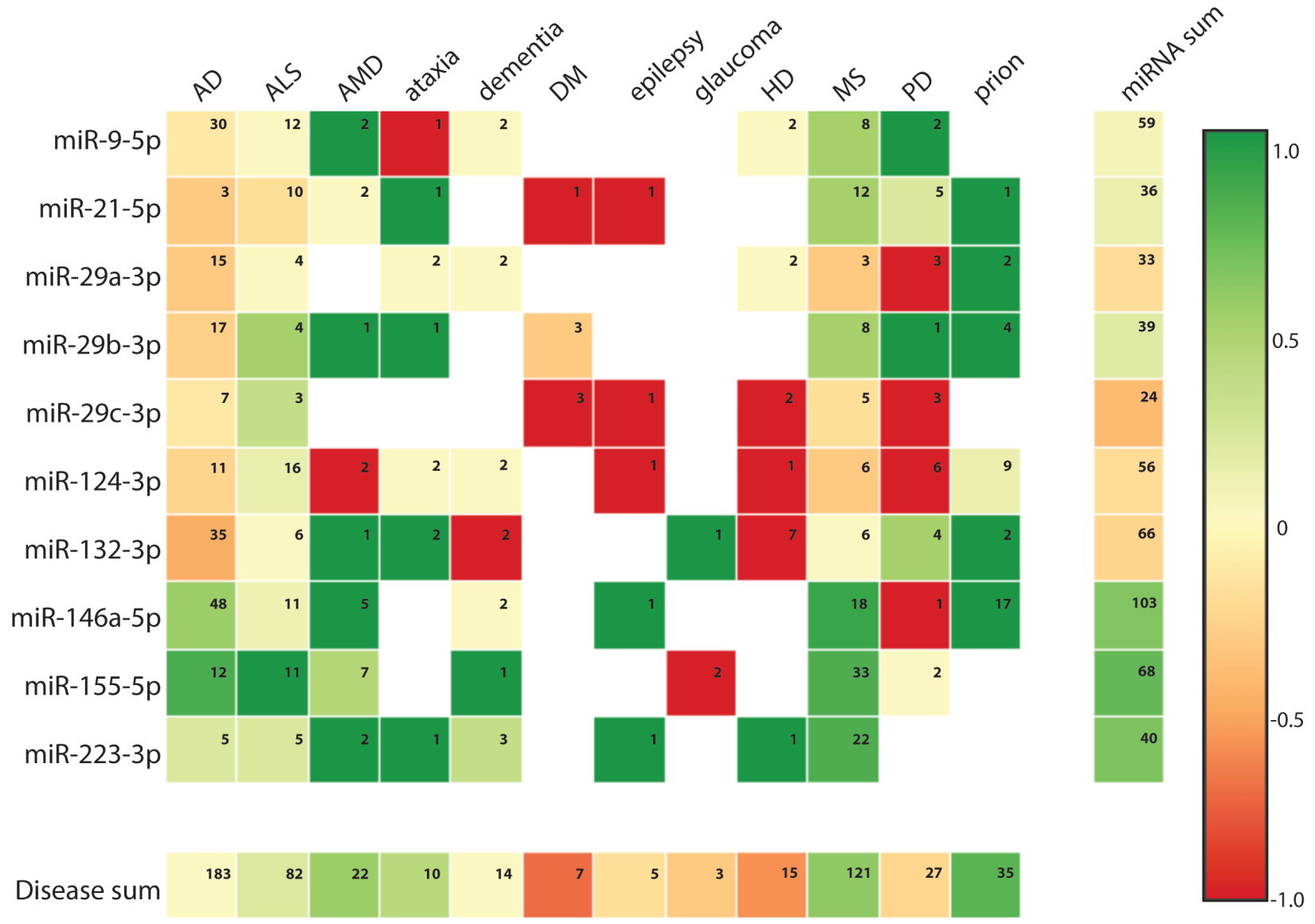

Figure 2. Heat map summary of the direction in which trending miRNAs are expressed across neurodegenerative disease. miRNAs with a rank of 1 were exclusively upregulated in a disease, -1 downregulated, and 0 implies the miRNA was equally upregulated and downregulated. Multiple values were taken from individual manuscripts when multiple time points and tissues were assessed. The number of values used to generate the heat map are listed within each rectangle. 
A

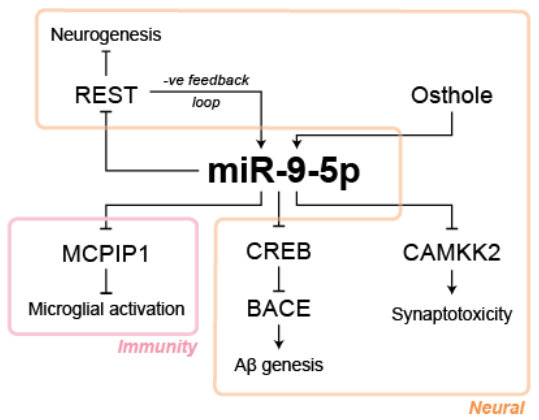

C

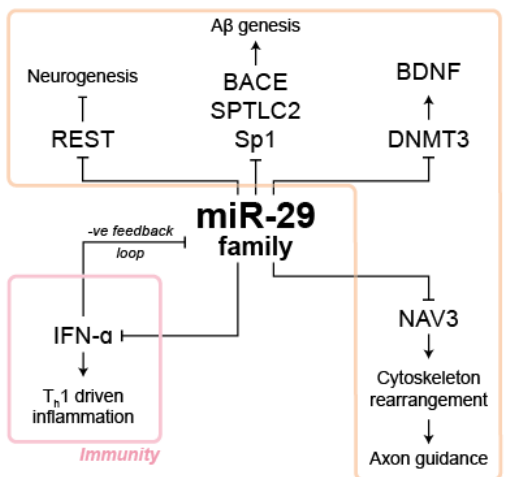

E

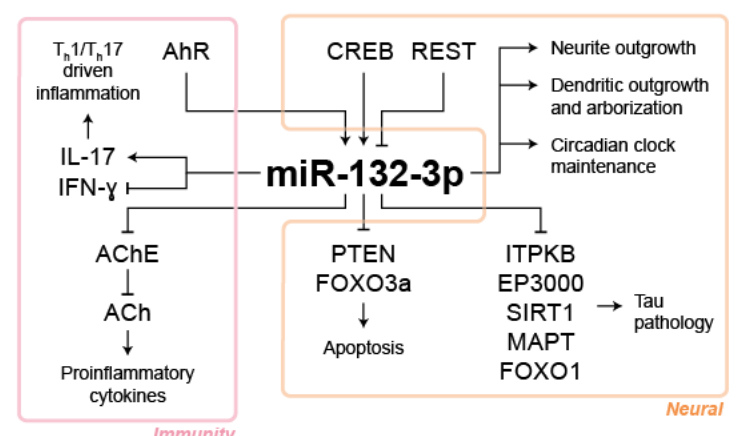

G

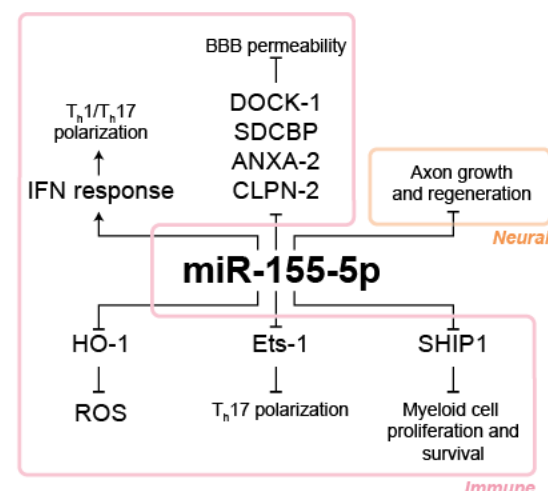

B

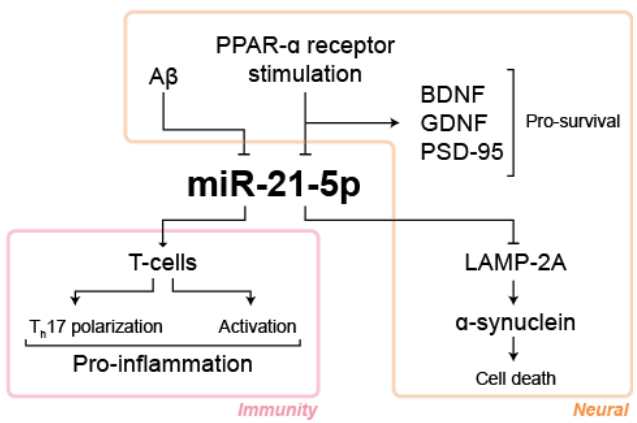

D

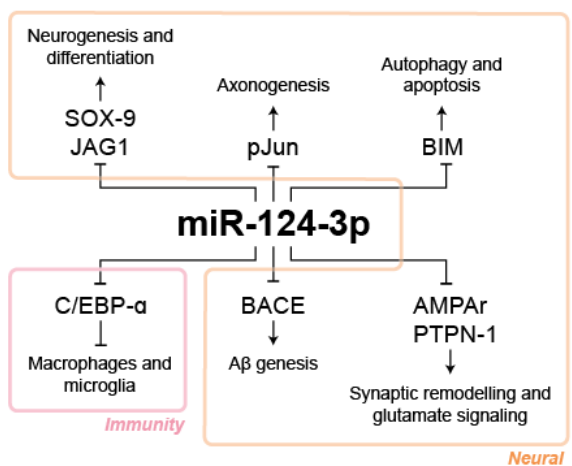

$\mathbf{F}$

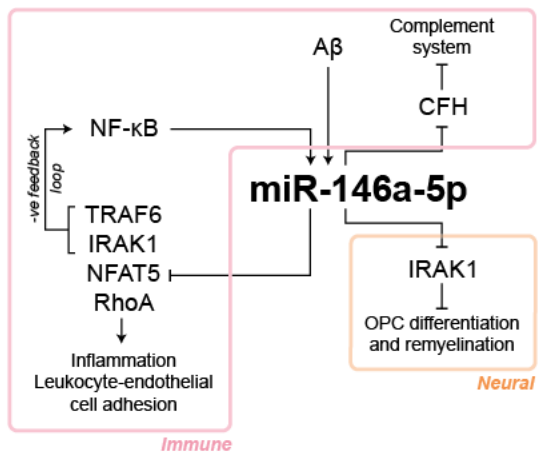

H

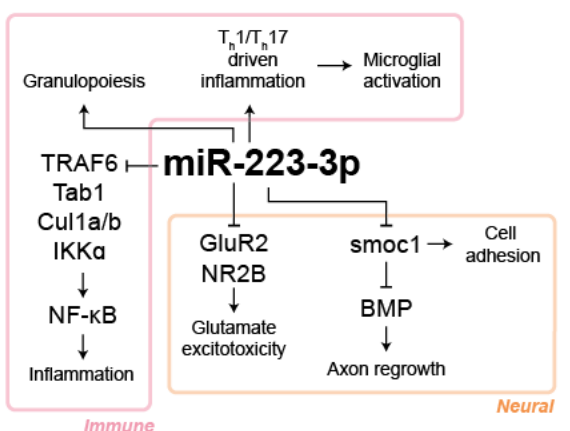

Figure 3. Summary flow chart of the neural and immune components regulated by key miRNAs identified as regulated across multiple neurodegenerative diseases. These miRNAs may act as underlying connections between the neural and immune components of neurodegenerative disease. The references for mRNA targets and/or specific signaling cascades are listed in Supplementary File 4. 


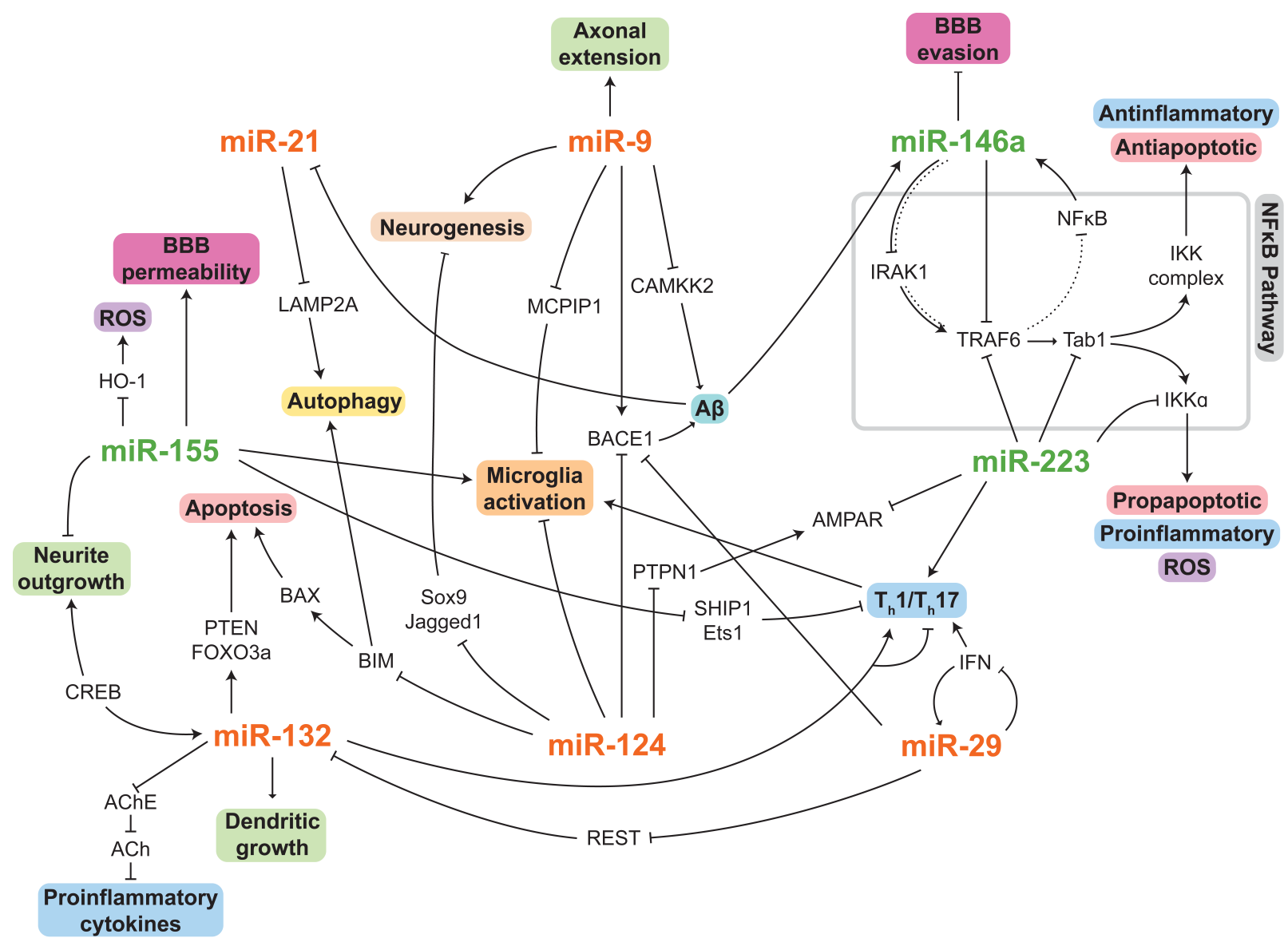

Figure 4. Shared pathways between commonly regulated miRNAs during neurodegenerative disease and their animal models. Overlaps include shared molecular pathways or differential pathways leading to similar end phenotypes, indicated by similarly coloured squares. miRNAs colour coded in green represent those that are generally upregulated during neurodegenerative disease; and orange represent those miRNAs with mixed regulation. Dotted arrows represent indirect interactions. 


\section{REFERENCES}

1. Bredesen, D.E., Rao, R.V. \& Mehlen, P. Cell death in the nervous system. Nature 443, 796-802 (2006).

2. Glass, C.K., Saijo, K., Winner, B., Marchetto, M.C. \& Gage, F.H. Mechanisms underlying inflammation in neurodegeneration. Cell 140, 918-934 (2010).

3. Ross, C.A. \& Poirier, M.A. Protein aggregation and neurodegenerative disease. Nat Med 10 Suppl, S10-17 (2004).

4. Dutta, R. \& Trapp, B.D. Mechanisms of neuronal dysfunction and degeneration in multiple sclerosis. Prog Neurobiol 93, 1-12 (2011).

5. Matias-Guiu, J.A. et al. Amyloid Proteins and Their Role in Multiple Sclerosis. Considerations in the Use of Amyloid-PeT imaging. Frontiers in Neurology 7 (2016).

6. Turner, P.R., O'Connor, K., Tate, W.P. \& Abraham, W.C. Roles of amyloid precursor protein and its fragments in regulating neural activity, plasticity and memory. Prog Neurobiol 70, 1-32 (2003).

7. Ellrichmann, G., Reick, C., Saft, C. \& Linker, R.A. The role of the immune system in Huntington's disease. Clin Dev Immunol 2013, 541259 (2013).

8. Colby, D.W. \& Prusiner, S.B. Prions. Cold Spring Harb Perspect Biol 3, a006833 (2011).

9. Strong, M.J. The evidence for altered RNA metabolism in amyotrophic lateral sclerosis (ALS). J Neurol Sci 288, 1-12 (2010).

10. Caillet-Boudin, M.L. et al. Brain pathology in myotonic dystrophy: when tauopathy meets spliceopathy and RNAopathy. Front Mol Neurosci 6, 57 (2014).

11. Orr, H.T. Cell biology of spinocerebellar ataxia. J Cell Biol 197, 167-177 (2012).

12. Dong, X.X., Wang, Y. \& Qin, Z.H. Molecular mechanisms of excitotoxicity and their relevance to pathogenesis of neurodegenerative diseases. Acta Pharmacol Sin 30, 379-387 (2009).

13. Mehta, A., Prabhakar, M., Kumar, P., Deshmukh, R. \& Sharma, P.L. Excitotoxicity: bridge to various triggers in neurodegenerative disorders. Eur J Pharmacol 698, 6-18 (2013).

14. Barker-Haliski, M. \& White, H.S. Glutamatergic Mechanisms Associated with Seizures and Epilepsy. Cold Spring Harb Perspect Med 5, a022863 (2015).

15. Kim, G.H., Kim, J.E., Rhie, S.J. \& Yoon, S. The Role of Oxidative Stress in Neurodegenerative Diseases. Exp Neurobiol 24, 325-340 (2015).

16. Shalgi, R., Lieber, D., Oren, M. \& Pilpel, Y. Global and local architecture of the mammalian microRNA-transcription factor regulatory network. PLoS Comput Biol 3, e131 (2007).

17. Nelson, P.T., Wang, W.X. \& Rajeev, B.W. MicroRNAs (miRNAs) in neurodegenerative diseases. Brain Pathol 18, 130-138 (2008).

18. Liang, H. \& Li, W.H. MicroRNA regulation of human protein protein interaction network. RNA 13, 1402-1408 (2007).

19. Lim, L.P. et al. Microarray analysis shows that some microRNAs downregulate large numbers of target mRNAs. Nature 433, 769-773 (2005).

20. Juźwik, C.A. et al. Neuronal microRNA regulation in Experimental Autoimmune Encephalomyelitis. Scientific Reports 8, 13437 (2018).

21. Ha, M. \& Kim, V.N. Regulation of microRNA biogenesis. Nat Rev Mol Cell Biol 15, 509-524 (2014).

22. Akhtar, S. et al. Histological Characterization of the Dicer1 Mutant Zebrafish Retina. J Ophthalmol 2015, 309510 (2015).

23. Hebert, S.S. et al. Genetic ablation of Dicer in adult forebrain neurons results in abnormal tau hyperphosphorylation and neurodegeneration. Hum Mol Genet 19, 3959-3969 (2010). 
24. lida, A., Shinoe, T., Baba, Y., Mano, H. \& Watanabe, S. Dicer plays essential roles for retinal development by regulation of survival and differentiation. Invest Ophthalmol Vis Sci 52, 30083017 (2011).

25. Cuellar, T.L. et al. Dicer loss in striatal neurons produces behavioral and neuroanatomical phenotypes in the absence of neurodegeneration. Proc Natl Acad Sci U S A 105, 5614-5619 (2008).

26. Damiani, D. et al. Dicer inactivation leads to progressive functional and structural degeneration of the mouse retina. J Neurosci 28, 4878-4887 (2008).

27. Chmielarz, P. et al. Dicer and microRNAs protect adult dopamine neurons. Cell Death Dis 8 , e2813 (2017).

28. Shin, D., Shin, J.Y., McManus, M.T., Ptacek, L.J. \& Fu, Y.H. Dicer ablation in oligodendrocytes provokes neuronal impairment in mice. Ann Neurol 66, 843-857 (2009).

29. Kaneko, H. et al. DICER1 deficit induces Alu RNA toxicity in age-related macular degeneration. Nature 471, 325-330 (2011).

30. Tao, J. et al. Deletion of astroglial Dicer causes non-cell-autonomous neuronal dysfunction and degeneration. J Neurosci 31, 8306-8319 (2011).

31. Fiorenza, A. et al. Blocking miRNA Biogenesis in Adult Forebrain Neurons Enhances Seizure Susceptibility, Fear Memory, and Food Intake by Increasing Neuronal Responsiveness. Cereb Cortex 26, 1619-1633 (2016).

32. Cheng, S. et al. Age-dependent neuron loss is associated with impaired adult neurogenesis in forebrain neuron-specific Dicer conditional knockout mice. Int J Biochem Cell Biol 57, 186-196 (2014).

33. Sundermeier, T.R. et al. MicroRNA-processing Enzymes Are Essential for Survival and Function of Mature Retinal Pigmented Epithelial Cells in Mice. J Biol Chem 292, 3366-3378 (2017).

34. Sundermeier, T.R. et al. DICER1 is essential for survival of postmitotic rod photoreceptor cells in mice. FASEB J 28, 3780-3791 (2014).

35. Aung, L.L. \& Balashov, K.E. Decreased Dicer expression is linked to increased expression of costimulatory molecule CD80 on B cells in multiple sclerosis. Multiple Sclerosis 21, 1131-1138 (2015).

36. Magner, W.J. et al. Dicer and microRNA expression in multiple sclerosis and response to interferon therapy. J Neuroimmunol 292, 68-78 (2016).

37. McKiernan, R.C. et al. Reduced mature microRNA levels in association with dicer loss in human temporal lobe epilepsy with hippocampal sclerosis. PLOS ONE [Electronic Resource] 7, e35921 (2012).

38. Lewkowicz, P. et al. Dysregulated RNA-Induced Silencing Complex (RISC) Assembly within CNS Corresponds with Abnormal miRNA Expression during Autoimmune Demyelination. Journal of Neuroscience 35, 7521-7537 (2015).

39. Henshall, D.C. et al. MicroRNAs in epilepsy: pathophysiology and clinical utility. The Lancet. Neurology 15, 1368-1376 (2016).

40. Swarbrick, S., Wragg, N., Ghosh, S. \& Stolzing, A. Systematic Review of miRNA as Biomarkers in Alzheimer's Disease. Molecular neurobiology (2019).

41. Dolati, S. et al. Dysregulated Network of miRNAs Involved in the Pathogenesis of Multiple Sclerosis. Biomedicine \& pharmacotherapy = Biomedecine \& pharmacotherapie 104, 280-290 (2018).

42. Plemel, J.R. et al. Over-the-counter anti-oxidant therapies for use in multiple sclerosis: A systematic review. Mult Scler 21, 1485-1495 (2015). 
43. Liberati, A. et al. The PRISMA statement for reporting systematic reviews and meta-analyses of studies that evaluate healthcare interventions: explanation and elaboration. BMJ 339, b2700 (2009).

44. Kozomara, A. \& Griffiths-Jones, S. miRBase: integrating microRNA annotation and deepsequencing data. Nucleic Acids Res 39, D152-157 (2011).

45. Plotly Collaborative data science Publisher: Plotly Technologies Inc. (2015).

46. Moore, C.S. et al. miR-155 as a multiple sclerosis-relevant regulator of myeloid cell polarization. Annals of Neurology 74, 709-720 (2013).

47. Butovsky, O. et al. Targeting miR-155 Restores Abnormal Microglia and Attenuates Disease in SOD1 Mice. Annals of Neurology 77, 75-99 (2015).

48. Thome, A.D., Harms, A.S., Volpicelli-Daley, L.A. \& Standaert, D.G. microRNA-155 Regulates Alpha-Synuclein-Induced Inflammatory Responses in Models of Parkinson Disease. Journal of Neuroscience 36, 2383-2390 (2016).

49. Ksiazek-Winiarek, D., Szpakowski, P., Turniak, M., Szemraj, J. \& Glabinski, A. IL-17 Exerts AntiApoptotic Effect via miR-155-5p Downregulation in Experimental Autoimmune Encephalomyelitis. Journal of Molecular Neuroscience 23, 23 (2017).

50. O'Connell, R.M. et al. MicroRNA-155 promotes autoimmune inflammation by enhancing inflammatory T cell development. Immunity 33, 607-619 (2010).

51. Murugaiyan, G., Beynon, V., Mittal, A., Joller, N. \& Weiner, H.L. Silencing microRNA-155 ameliorates experimental autoimmune encephalomyelitis. Journal of Immunology 187, 22132221 (2011).

52. Zhang, J. \& Braun, M.Y. PD-1 deletion restores susceptibility to experimental autoimmune encephalomyelitis in miR-155-deficient mice. International Immunology 26, 407-415 (2014).

53. Zhang, J. \& Braun, M.Y. Protoporphyrin Treatment Modulates Susceptibility to Experimental Autoimmune Encephalomyelitis in miR-155-Deficient Mice. PLoS ONE [Electronic Resource] 10, e0145237 (2015).

54. Zhang, J. et al. MicroRNA-155 modulates Th1 and Th17 cell differentiation and is associated with multiple sclerosis and experimental autoimmune encephalomyelitis. Journal of Neuroimmunology 266, 56-63 (2014).

55. Mycko, M.P., Cichalewska, M., Cwiklinska, H. \& Selmaj, K.W. miR-155-3p Drives the Development of Autoimmune Demyelination by Regulation of Heat Shock Protein 40. Journal of Neuroscience 35, 16504-16515 (2015).

56. Hu, R. et al. MicroRNA-155 confers encephalogenic potential to Th17 cells by promoting effector gene expression. Journal of Immunology 190, 5972-5980 (2013).

57. Koval, E.D. et al. Method for widespread microRNA-155 inhibition prolongs survival in ALSmodel mice. Human Molecular Genetics 22, 4127-4135 (2013).

58. Lopez-Ramirez, M.A. et al. MicroRNA-155 negatively affects blood-brain barrier function during neuroinflammation. FASEB Journal 28, 2551-2565 (2014).

59. Johnnidis, J.B. et al. Regulation of progenitor cell proliferation and granulocyte function by microRNA-223. Nature 451, 1125-1129 (2008).

60. Fazi, F. et al. A minicircuitry comprised of microRNA-223 and transcription factors NFI-A and C/EBPalpha regulates human granulopoiesis. Cell 123, 819-831 (2005).

61. Chen, C.Z., Li, L., Lodish, H.F. \& Bartel, D.P. MicroRNAs modulate hematopoietic lineage differentiation. Science 303, 83-86 (2004).

62. Ifergan, I., Chen, S., Zhang, B. \& Miller, S.D. Cutting Edge: MicroRNA-223 Regulates Myeloid Dendritic Cell-Driven Th17 Responses in Experimental Autoimmune Encephalomyelitis. J Immunol 196, 1455-1459 (2016). 
63. Satoorian, T. et al. MicroRNA223 promotes pathogenic T-cell development and autoimmune inflammation in central nervous system in mice. Immunology 148, 326-338 (2016).

64. Cantoni, C. et al. Mir-223 regulates the number and function of myeloid-derived suppressor cells in multiple sclerosis and experimental autoimmune encephalomyelitis. Acta Neuropathol 133, 61-77 (2017).

65. Li, T. et al. MicroRNAs modulate the noncanonical transcription factor NF-kappaB pathway by regulating expression of the kinase IKKalpha during macrophage differentiation. Nat Immunol 11, 799-805 (2010).

66. Zhou, W. et al. MicroRNA-223 Suppresses the Canonical NF-kappaB Pathway in Basal Keratinocytes to Dampen Neutrophilic Inflammation. Cell Rep 22, 1810-1823 (2018).

67. Harraz, M.M., Eacker, S.M., Wang, X., Dawson, T.M. \& Dawson, V.L. MicroRNA-223 is neuroprotective by targeting glutamate receptors. Proc Natl Acad Sci U S A 109, 18962-18967 (2012).

68. Morquette, B. et al. MicroRNA-223 protects neurons from degeneration in Experimental Autoimmune Encephalomyelitis. bioRxiv (2018).

69. Strickland, I.T. et al. Axotomy-induced miR-21 promotes axon growth in adult dorsal root ganglion neurons. PLOS ONE 6, e23423 (2011).

70. Fuller-Carter, P.I. et al. Integrated analyses of zebrafish miRNA and mRNA expression profiles identify miR-29b and miR-223 as potential regulators of optic nerve regeneration. $B M C$ Genomics 16, 591 (2015).

71. Iyer, A. et al. MicroRNA-146a: a key regulator of astrocyte-mediated inflammatory response. PLoS One 7, e44789 (2012).

72. Boldin, M.P. et al. miR-146a is a significant brake on autoimmunity, myeloproliferation, and cancer in mice. J Exp Med 208, 1189-1201 (2011).

73. Li, B. et al. miR-146a modulates autoreactive Th17 cell differentiation and regulates organspecific autoimmunity. J Clin Invest 127, 3702-3716 (2017).

74. Wu, D. et al. Brain endothelial miR-146a negatively modulates T-cell adhesion through repressing multiple targets to inhibit NF-kappaB activation. J Cereb Blood Flow Metab 35, 412423 (2015).

75. Zhang, J. et al. MiR-146a promotes remyelination in a cuprizone model of demyelinating injury. Neuroscience 348, 252-263 (2017).

76. Cui, J.G., Li, Y.Y., Zhao, Y., Bhattacharjee, S. \& Lukiw, W.J. Differential regulation of interleukin-1 receptor-associated kinase-1 (IRAK-1) and IRAK-2 by microRNA-146a and NF-kappaB in stressed human astroglial cells and in Alzheimer disease. J Biol Chem 285, 38951-38960 (2010).

77. Li, Y.Y. et al. Increased expression of miRNA-146a in Alzheimer's disease transgenic mouse models. Neurosci Lett 487, 94-98 (2011).

78. Kempf, S.J. et al. An integrated proteomics approach shows synaptic plasticity changes in an APP/PS1 Alzheimer's mouse model. Oncotarget 7, 33627-33648 (2016).

79. Gerrard, B. et al. Chronic mild stress exacerbates severity of experimental autoimmune encephalomyelitis in association with altered non-coding RNA and metabolic biomarkers. Neuroscience 359, 299-307 (2017).

80. Zhao, C., Sun, G., Li, S. \& Shi, Y. A feedback regulatory loop involving microRNA-9 and nuclear receptor TLX in neural stem cell fate determination. Nat Struct Mol Biol 16, 365-371 (2009).

81. Tan, S.L., Ohtsuka, T., Gonzalez, A. \& Kageyama, R. MicroRNA9 regulates neural stem cell differentiation by controlling Hes1 expression dynamics in the developing brain. Genes Cells 17, 952-961 (2012).

82. Lagos-Quintana, M. et al. Identification of tissue-specific microRNAs from mouse. Curr Biol 12, 735-739 (2002). 
83. Chi, L. et al. Motor neuron degeneration promotes neural progenitor cell proliferation, migration, and neurogenesis in the spinal cords of amyotrophic lateral sclerosis mice. Stem Cells 24, 34-43 (2006).

84. Guan, Y.J. et al. Increased stem cell proliferation in the spinal cord of adult amyotrophic lateral sclerosis transgenic mice. J Neurochem 102, 1125-1138 (2007).

85. Marcuzzo, S. et al. Up-regulation of neural and cell cycle-related microRNAs in brain of amyotrophic lateral sclerosis mice at late disease stage. Mol Brain 8, 5 (2015).

86. Marcuzzo, S. et al. Altered miRNA expression is associated with neuronal fate in G93A-SOD1 ependymal stem progenitor cells. Exp Neurol 253, 91-101 (2014).

87. Zhou, F.H. et al. miRNA-9 expression is upregulated in the spinal cord of G93A-SOD1 transgenic mice. International Journal of Clinical and Experimental Pathology 6, 1826-1838 (2013).

88. Packer, A.N., Xing, Y., Harper, S.Q., Jones, L. \& Davidson, B.L. The bifunctional microRNA miR9/miR-9* regulates REST and CoREST and is downregulated in Huntington's disease. $J$ Neurosci 28, 14341-14346 (2008).

89. Danilov, A.l. et al. Neurogenesis in the adult spinal cord in an experimental model of multiple sclerosis. Eur J Neurosci 23, 394-400 (2006).

90. Chang, A. et al. Neurogenesis in the chronic lesions of multiple sclerosis. Brain 131, 2366-2375 (2008).

91. Winner, B. \& Winkler, J. Adult neurogenesis in neurodegenerative diseases. Cold Spring Harb Perspect Biol 7, a021287 (2015).

92. Winner, B., Kohl, Z. \& Gage, F.H. Neurodegenerative disease and adult neurogenesis. Eur J Neurosci 33, 1139-1151 (2011).

93. Su, C., Yang, X. \& Lou, J. Geniposide reduces alpha-synuclein by blocking microRNA21/lysosome-associated membrane protein $2 A$ interaction in Parkinson disease models. Brain Res 1644, 98-106 (2016).

94. Murugaiyan, G. et al. MicroRNA-21 promotes Th17 differentiation and mediates experimental autoimmune encephalomyelitis. J Clin Invest 125, 1069-1080 (2015).

95. Bergman, P. et al. Next-generation sequencing identifies microRNAs that associate with pathogenic autoimmune neuroinflammation in rats. Journal of Immunology 190, 4066-4075 (2013).

96. Fu, Y., Zhen, J. \& Lu, Z. Synergetic Neuroprotective Effect of Docosahexaenoic Acid and Aspirin in SH-Y5Y by Inhibiting miR-21 and Activating RXRalpha and PPARalpha. DNA Cell Biol 36, 482-489 (2017).

97. Wang, W.X., Huang, Q., Hu, Y., Stromberg, A.J. \& Nelson, P.T. Patterns of microRNA expression in normal and early Alzheimer's disease human temporal cortex: white matter versus gray matter. Acta Neuropathologica 121, 193-205 (2011).

98. Burgos, K. et al. Profiles of extracellular miRNA in cerebrospinal fluid and serum from patients with Alzheimer's and Parkinson's diseases correlate with disease status and features of pathology. PLoS ONE [Electronic Resource] 9, e94839 (2014).

99. Weinberg, R.B., Mufson, E.J. \& Counts, S.E. Evidence for a neuroprotective microRNA pathway in amnestic mild cognitive impairment. Frontiers in Neuroscience 9 (NOV) (no pagination) (2015).

100. Schonrock, N. et al. Neuronal microRNA deregulation in response to Alzheimer's disease amyloid-beta. PLoS One 5, e11070 (2010).

101. Cui, G.H. et al. Exosomes derived from hypoxia-preconditioned mesenchymal stromal cells ameliorate cognitive decline by rescuing synaptic dysfunction and regulating inflammatory responses in APP/PS1 mice. FASEB J 32, 654-668 (2018).

102. Su, W. et al. microRNA-21a-5p/PDCD4 axis regulates mesenchymal stem cell-induced neuroprotection in acute glaucoma. J Mol Cell Biol 9, 289-301 (2017). 
103. Guo, L. et al. Targeting amyloid-beta in glaucoma treatment. Proc Natl Acad Sci U S A 104, 13444-13449 (2007).

104. Smith, K.M. et al. miR-29ab1 deficiency identifies a negative feedback loop controlling Th1 bias that is dysregulated in multiple sclerosis. Journal of Immunology 189, 1567-1576 (2012).

105. Papadopoulou, A.S. et al. Deficiency of the miR-29a/b-1 cluster leads to ataxic features and cerebellar alterations in mice. Neurobiology of Disease 73, 275-288 (2015).

106. Roshan, R. et al. Brain-specific knockdown of miR-29 results in neuronal cell death and ataxia in mice. Rna-A Publication of the Rna Society 20, 1287-1297 (2014).

107. Serafin, A. et al. Overexpression of blood microRNAs 103a, 30b, and 29a in L-dopa-treated patients with PD. Neurology 84, 645-653 (2015).

108. Schwienbacher, C. et al. Plasma and White Blood Cells Show Different miRNA Expression Profiles in Parkinson's Disease. J Mol Neurosci 62, 244-254 (2017).

109. Ingwersen, J. et al. Natalizumab restores aberrant miRNA expression profile in multiple sclerosis and reveals a critical role for miR-20b. Annals of Clinical \& Translational Neurology 2, 43-55 (2015).

110. Ma, F. et al. The microRNA miR-29 controls innate and adaptive immune responses to intracellular bacterial infection by targeting interferon-gamma. Nat Immunol 12, 861-869 (2011).

111. Hecker, M. et al. MicroRNA expression changes during interferon-beta treatment in the peripheral blood of multiple sclerosis patients. International Journal of Molecular Sciences 14, 16087-16110 (2013).

112. Kong, Y., Wu, J., Zhang, D., Wan, C. \& Yuan, L. The role of miR-124 in Drosophila Alzheimer's disease by targeting delta in notch signalling pathway. Current Molecular Medicine 15, 980-989 (2015).

113. Du, X. et al. miR-124 downregulates BACE 1 and alters autophagy in APP/PS1 transgenic mice. Toxicol Lett 280, 195-205 (2017).

114. Saraiva, C., Paiva, J., Santos, T., Ferreira, L. \& Bernardino, L. MicroRNA-124 loaded nanoparticles enhance brain repair in Parkinson's disease. J Control Release 235, 291-305 (2016).

115. Ponomarev, E.D., Veremeyko, T., Barteneva, N., Krichevsky, A.M. \& Weiner, H.L. MicroRNA-124 promotes microglia quiescence and suppresses EAE by deactivating macrophages via the C/EBPalpha-PU.1 pathway. Nat Med 17, 64-70 (2011).

116. Liu, T., Im, W., Mook-Jung, I. \& Kim, M. MicroRNA-124 slows down the progression of Huntington's disease by promoting neurogenesis in the striatum. Neural Regen Res 10, 786-791 (2015).

117. He, Y. et al. MiR-124 Promotes the Growth of Retinal Ganglion Cells Derived from Muller Cells. Cell Physiol Biochem 45, 973-983 (2018).

118. Chu-Tan, J.A. et al. MicroRNA-124 Dysregulation is Associated With Retinal Inflammation and Photoreceptor Death in the Degenerating Retina. Invest Ophth Vis Sci 59, 4094-4105 (2018).

119. Zhou, Y., Deng, J., Chu, X., Zhao, Y. \& Guo, Y. Role of Post-Transcriptional Control of Calpain by miR-124-3p in the Development of Alzheimer's Disease. J Alzheimers Dis 67, 571-581 (2019).

120. Yao, L. et al. MicroRNA-124 regulates the expression of MEKK3 in the inflammatory pathogenesis of Parkinson's disease. J Neuroinflammation 15, 13 (2018).

121. Wang, H. et al. MiR-124 Regulates Apoptosis and Autophagy Process in MPTP Model of Parkinson's Disease by Targeting to Bim. Brain Pathol 26, 167-176 (2016).

122. Wang, X. et al. A Novel MicroRNA-124/PTPN1 Signal Pathway Mediates Synaptic and Memory Deficits in Alzheimer's Disease. Biol Psychiatry 83, 395-405 (2018).

123. Gascon, E. et al. Alterations in microRNA-124 and AMPA receptors contribute to social behavioral deficits in frontotemporal dementia. Nat Med 20, 1444-1451 (2014). 
124. Dutta, R. et al. Hippocampal demyelination and memory dysfunction are associated with increased levels of the neuronal microRNA miR-124 and reduced AMPA receptors. Ann Neurol 73, 637-645 (2013).

125. Smith, P.Y. et al. miR-132/212 deficiency impairs tau metabolism and promotes pathological aggregation in vivo. Human Molecular Genetics 24, 6721-6735 (2015).

126. Shaked, I. et al. MicroRNA-132 potentiates cholinergic anti-inflammatory signaling by targeting acetylcholinesterase. Immunity 31, 965-973 (2009).

127. Vo, N. et al. A cAMP-response element binding protein-induced microRNA regulates neuronal morphogenesis. Proc Natl Acad Sci U S A 102, 16426-16431 (2005).

128. Magill, S.T. et al. microRNA-132 regulates dendritic growth and arborization of newborn neurons in the adult hippocampus. Proc Natl Acad Sci U S A 107, 20382-20387 (2010).

129. Cheng, H.Y. et al. microRNA modulation of circadian-clock period and entrainment. Neuron 54, 813-829 (2007).

130. Wong, H.K. et al. De-repression of FOXO3a death axis by microRNA-132 and -212 causes neuronal apoptosis in Alzheimer's disease. Human Molecular Genetics 22, 3077-3092 (2013).

131. Salta, E., Sierksma, A., Vanden Eynden, E. \& De Strooper, B. miR-132 loss de-represses ITPKB and aggravates amyloid and TAU pathology in Alzheimer's brain. EMBO Molecular Medicine. (2016).

132. Lau, P. et al. Alteration of the microRNA network during the progression of Alzheimer's disease. EMBO molecular medicine 5, 1613-1634 (2013).

133. Hanieh, H. \& Alzahrani, A. MicroRNA-132 suppresses autoimmune encephalomyelitis by inducing cholinergic anti-inflammation: a new Ahr-based exploration. European Journal of Immunology 43, 2771-2782 (2013).

134. Nakahama, T. et al. Aryl hydrocarbon receptor-mediated induction of the microRNA-132/212 cluster promotes interleukin-17-producing T-helper cell differentiation. Proceedings of the National Academy of Sciences of the United States of America 110, 11964-11969 (2013).

135. Lykhmus, O. et al. Molecular Mechanisms Regulating LPS-Induced Inflammation in the Brain. Frontiers in Molecular Neuroscience 9, 19 (2016).

136. Mameli, G. et al. Natalizumab Therapy Modulates miR-155, miR-26a and Proinflammatory Cytokine Expression in MS Patients. PLoS ONE [Electronic Resource] 11, e0157153 (2016).

137. Miyazaki, Y. et al. A novel microRNA-132-sirtuin-1 axis underlies aberrant B-cell cytokine regulation in patients with relapsing-remitting multiple sclerosis [corrected].[Erratum appears in PLoS One. 2014;9(9):e109041]. PLoS ONE [Electronic Resource] 9, e105421 (2014).

138. Flamand, M.N., Gan, H.H., Mayya, V.K., Gunsalus, K.C. \& Duchaine, T.F. A non-canonical site reveals the cooperative mechanisms of microRNA-mediated silencing. Nucleic Acids Res 45, 7212-7225 (2017).

139. Schmitz, U. et al. Cooperative gene regulation by microRNA pairs and their identification using a computational workflow. Nucleic Acids Res 42, 7539-7552 (2014).

140. Hayden, M.S., West, A.P. \& Ghosh, S. NF-kappaB and the immune response. Oncogene 25, 67586780 (2006).

141. Srinivasan, M. \& Lahiri, D.K. Significance of NF-kappa B as a pivotal therapeutic target in the neurodegenerative pathologies of Alzheimer's disease and multiple sclerosis. Expert Opinion on Therapeutic Targets 19, 471-487 (2015).

142. Tang, Y. \& Le, W. Differential Roles of M1 and M2 Microglia in Neurodegenerative Diseases. Mol Neurobiol 53, 1181-1194 (2016).

143. Ginhoux, F., Lim, S., Hoeffel, G., Low, D. \& Huber, T. Origin and differentiation of microglia. Front Cell Neurosci 7, 45 (2013).

144. Soreq, H. \& Wolf, Y. NeurimmiRs: microRNAs in the neuroimmune interface. Trends Mol Med 17, 548-555 (2011). 
